\title{
$A b$ initio study of optical absorption spectra of semiconductors and conjugated polymers
}

\author{
M. L. Tiago ${ }^{1}$, Eric K. Chang ${ }^{1}$, Michael Rohlfing ${ }^{2}$, and Steven G. Louie ${ }^{1}$ \\ ${ }^{1}$ Department of Physics, University of California at Berkeley, Berkeley, CA, 94720, USA and Materials Science Division , \\ Lawrence Berkeley National Laboratory, Berkeley, CA, 94720 \\ ${ }^{2}$ Institut für Theoretische Physik II - Festkörperphysik, Universität Münster, Wilhelm-Klemm-Straße 10, 48149 Münster, \\ Germany
}

\begin{abstract}
The effects of electron-hole interaction on the optical properties of a variety of materials have been calculated using an $a b$ initio method based on solving the BetheSalpeter equation. Results on selected semiconductors, insulators, and semiconducting polymers are presented. In the cases of alpha-quartz $\left(\mathrm{SiO}_{2}\right)$ and poly-phenylene-vinylene, resonant excitonic states qualitatively alter the absorption spectra.
\end{abstract}

\section{Introduction}

The role of electron-hole interactions in the optical properties of semiconductors and insulators has been long recognized (see e.g. [1]). It is possible now to get an accurate description of such properties for a large class of materials from first principles calculations [2-4]. The theoretical framework is based on solving a Bethe-Salpeter equation for the electron-hole amplitude of the 2-particle Green's function[5]. Our approach [2] takes into account electron self energy effects and the interaction between an optically excited electron and the hole left behind. The underlying one-particle (quasiparticle) description is taken within the GW approximation for the electron self-energy [6].

\section{Theory}

In moderately correlated electron systems, the photoexcited states can be written, to a good approximation, as a linear combination of free electron-hole configurations,

$|S\rangle=\sum_{c v \mathbf{k}} A_{c v \mathbf{k}}^{S}|c \mathbf{k} ; v \mathbf{k}\rangle$

where $|c \mathbf{k} ; v \mathbf{k}\rangle$ represents a configuration in which a quasielectron is promoted from the valence band $v$ to the conduction band $c$. Only vertical transitions are taken into account. From the Bethe-Salpeter equation for the twoparticle Green's function, the coefficients $A_{c v k}^{S}$ satisfy an eigenvalue equation,

$$
\left(E_{c, \mathbf{k}}-E_{v, \mathbf{k}}\right) A_{c v \mathbf{k}}^{S}+\sum_{c v \mathbf{k}, c^{\prime} v^{\prime} \mathbf{k}^{\prime}} \mathcal{K}_{c v \mathbf{k}}^{c^{\prime} v^{\prime} \mathbf{k}^{\prime}} A_{c^{\prime} v^{\prime} \mathbf{k}^{\prime}}^{S}=\Omega^{S} A_{c v \mathbf{k}}^{S}
$$

where $\mathcal{K}_{c v \mathbf{k}}^{c^{\prime} v^{\prime} \mathbf{k}^{\prime}}$ describes the interaction between the excited electron and hole and $\Omega^{S}$ is the energy of state $|S\rangle$. In general, $\mathcal{K}$ is a complicated function that describes the scattering of an electron-hole pair in configuration $|c \mathbf{k} ; v \mathbf{k}\rangle$ to $\left|c^{\prime} \mathbf{k}^{\prime} ; v^{\prime} \mathbf{k}^{\prime}\right\rangle$. This can be simplified to a screened attractive interaction plus a bare repulsive ex-
Fig. 1 Absorption spectrum of bulk silicon, with (full line) and without (dotted-dashed line) excitonic effects. The experimental spectrum (dots) is from [7].

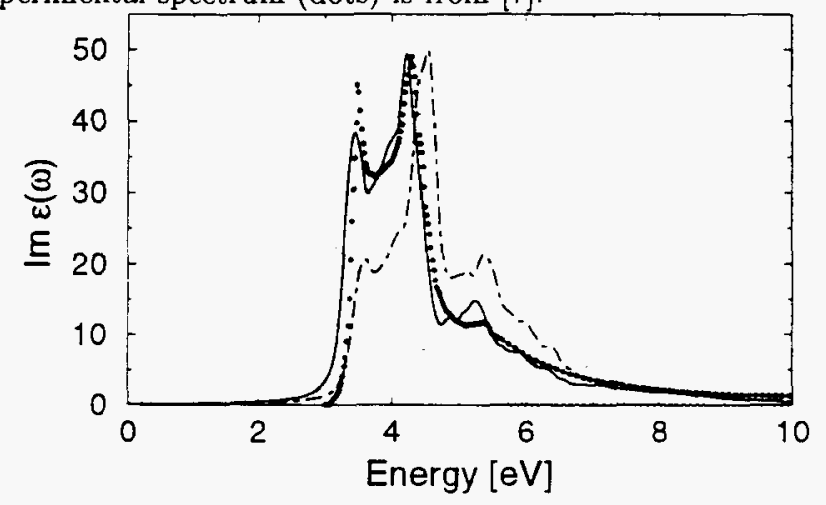

change interaction [2]. Dynamical interactions can also be neglected if the energies $\Omega^{S}$ are close to the energy of non-interacting pairs.

The optical absorption is given by

$\epsilon_{2}(\omega)=\frac{4 \pi^{2} e^{2}}{\omega^{2}} \frac{1}{V_{c}} \sum_{S}|\langle G|\lambda \cdot \mathbf{v}| S\rangle|^{2} \delta\left(\Omega_{S}-\hbar \omega\right)$

where $|G\rangle$ is the ground state and $\lambda$ and $\mathrm{v}$ are the polarization vector and the velocity operator, respectively.

The one-particle energies in Eq. (2) are taken from a GWA calculation, as described by Hybertsen and Louie [6].

\section{Applications}

The measured absorption spectrum of silicon, plotted in Figure 1, shows two well-pronounced peaks at energies about $3.5 \mathrm{eV}$ and $4.2 \mathrm{eV}$ [7]. The calculated results using Eq. (3) reproduce the experimental data very accurately. The minor discrepancies at the energy range 5.5-6.0 $\mathrm{eV}$ may be due to the finite sampling of points in the Brillouin zone. The large enhancement in the amplitude of the first peak is due to excitonic effects.

The optical properties of alpha quartz $\left(\mathrm{SiO}_{2}\right)$ have been a subject of debate since the 1960's [8]. The presence of a series of four sharp peaks in its absorption spectrum has been recently shown to be due to the formation of resonant excitons [3].

In Figure 2, we show the optical absorption of $\mathrm{SiO}_{2}$ for polarization perpendicular to the hexagonal plane. The agreement between the experimental spectrum (with peaks at energies 10.3, 11.7, 14.0 and $17.3 \mathrm{eV}$ ) and the 
Fig. 2 Absorption spectrum of alpha quartz $\left(\mathrm{SiO}_{2}\right)$ with excitonic effects (solid line) as compared to the interband transition theory (dotted-dashed line). Experimental data are given by the dashed line [8].

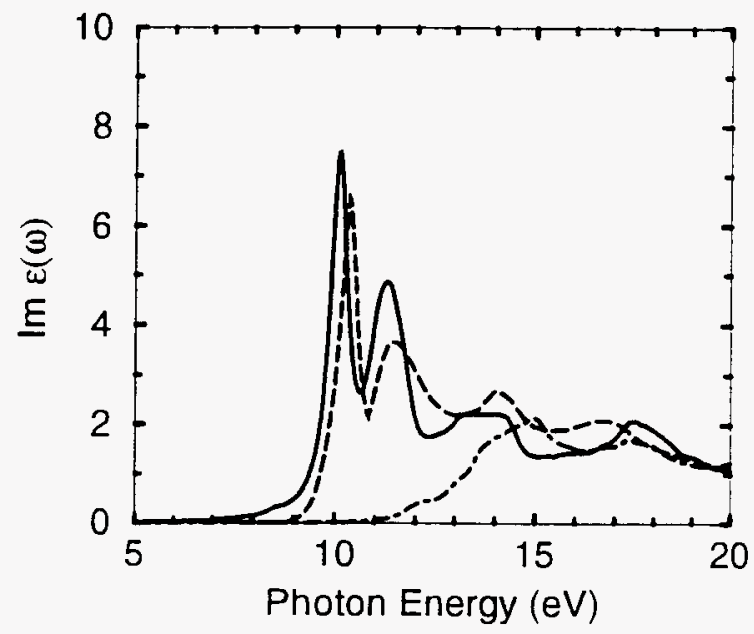

Table 1 Calculated binding energies of excitons in GaAs, compared with data from optical absorption [9] and from two-photon absorption [10].

\begin{tabular}{ccc}
\hline & this work [meV] & Exp. [meV] \\
\hline$E_{1 s}$ & 4.0 & $4.2^{a}$ \\
$E_{2 s}$ & 0.9 & $1.09^{a}$ \\
$E_{1 p}$ & $0.2-0.7$ & $\sim 0.1^{b}$ \\
\hline
\end{tabular}

${ }^{a}$ Reference [9]

${ }^{b}$ Reference [10]

calculated spectrum, with excitonic effects included, is again excellent. The fact that an interband transition description gives an almost featureless absorption spectrum shows that the formation of optically active resonant excitonic states dominates the spectrum up to energies about $18 \mathrm{eV}$.

It is also possible to obtain the binding energy of bound excitons very accurately from Eq. (2), even when this binding energy is only a small fraction of $\mathrm{eV}$. In this case, a careful sampling of the Brillouin zone is required, so that the electronic energy bands are described with the necessary accuracy. Table 1 shows the binding energy of the lowest energy excitonic states in GaAs [2]. In this calculation, we made use of a set of 1000 special k-points near the position of the band extrema.

As an example of a lower-dimensional system, we present results for the conjugated polymer, poly-phenylenevinylene (PPV), in Figure 3. The difference between the results with and without excitonic effects is striking. The peak at $2.4 \mathrm{eV}$ results from coherent coupled transition$\mathrm{s}$ between the highest occupied band and lowest unoccupied band. The resulting exciton has a large binding energy of $0.9 \mathrm{eV}$. The measured absorption peaks are located at energies 2.5, 3.7, 4.8 and $6.0 \mathrm{eV}$, which are in quite good agreement with the calculated peak posi-
Fig. 3 Calculated optical absorption spectrum of PPV, with (solid line) and without (dashed line) excitonic effects. An artificial broadening of $0.05 \mathrm{eV}$ is included, and the polarization vector is along the chain.

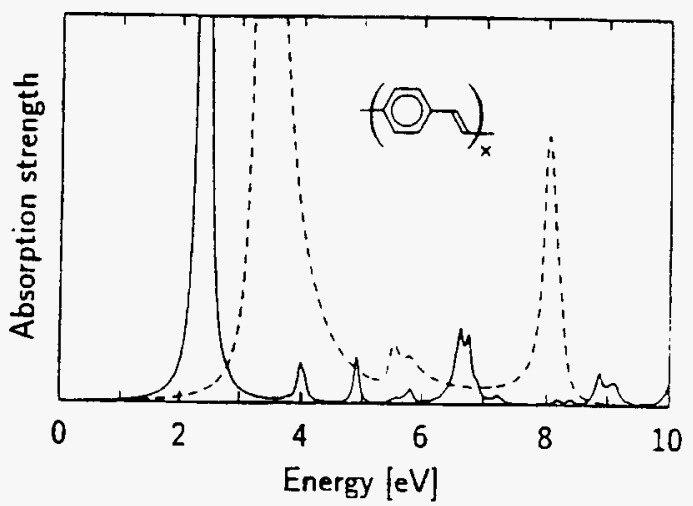

tions. As in the case of $\mathrm{SiO}_{2}$, the spectrum of PPV is dominated by resonant excitons.

\section{Conclusion}

In summary, we have shown that an $a b$ initio method, based on solving the Bethe-Salpeter equation, is capable of describing successfully the optical properties of a variety of semiconductors and insulators. The agreement with experimental data is remarkably good. The same analysis can be and have been applied to other systems including surfaces and clusters[2].

\section{Acknowledgments}

This work was supported by NSF Grant No. DMR-952055 U.S. Department of Energy under Contract No. DEAC03-76SF00098, and by the Deustche Forschungsgemeinschaft (Bonn, Germany) under Grant No. Ro-1318/11. Computational resources were provided by NERSC and by the NSF.

\section{References}

1. F. Bassani and G. Pastori Parravicini, Electronic States and Optical Transitions in Solids (Pergamon Press. Oxford 1975)

2. M. Rohlfing and S. G. Louie. Phys. Rev. Lett. 81, 2312 (1998); Phys. Rev. B, 62, 4927 (2000)

3. E. K. Chang, M. Rohlfing and S. G. Louie, to appear in Phys. Rev. Lett.

4. L. X. Benedict, E. L. Shirley and R. B. Bohn, Phys. Rev. Lett. 80, 4514 (1998) ; Phys. Rev. B 59, 5441 (1999) ; S. Albrecht, L. Reining, R. Del Sole and G. Onida, Phys. Rev. Lett. 80, 4510 (1998)

5. G. Strinati, Phys. Rev. B 29, 5718 (1984)

6. M. S. Hybertsen and S. G. Louie, Phys. Rev. Let. 55, 1418 (1985); Phys. Rev. B 34, 5390 (1986)

7. D. E. Aspnes and A. A. Studna, Phys. Rev B 27, 985 (1983)

8. H. R. Philipp, Solid State Commun., 4, 73 (1966)

9. D. D. Sell, Phys. Rev. B 6, 3750 (1972)

10. J. S. Michaelis, K. Unterrainer, E. Gornik, and E. Bauser,

Phys. Rev B 54, 7917 (1996)

11. S. Mukamel et al., Science 277, 781 (1997) 